\title{
THE PROTEUS APPROACH OF MAINTENANCE WORK FLOW MANAGEMENT
}

\author{
Mario Thron, Thomas Bangemann \\ ifak Institut für Automation und Kommunikation e.V. Magdeburg \\ Steinfeldstr. 3, D-39179 Barleben, Germany
}

\begin{abstract}
Equipment maintenance is an essential need for successful industrial enterprises. Several software applications are used today for initiation and management of maintenance activities. This paper introduces the PROTEUS integration platform for cooperative interactions between maintenance applications. It is necessary to define the order of these interactions, the so-called business logic workflow. The paper shows the approach of workflow management starting from user-defined scenarios leading to runtime implementations for the PROTEUS platform. Copyright $\odot 2005$ IFAC
\end{abstract}

Keywords: E-maintenance, concurrent engineering, service integration, Web Service, workflow programming.

\section{INTRODUCTION}

The origin goals of industrial enterprises are to manufacture and to sell products. The efficiency of the production process is a key factor for the profitability of the enterprise. A variety of activities has to be managed in the production process as well as in the preparation and post processing phase. Examples of these activities are shown in Figure 1.

These activities itself have been studied and optimised for a long time with the main objective of an improvement of the profits. Benefits come from several reasons like optimality and novelty of product design and quality, increasing productivity of the production process, financial optimum investments and further more.

Optimisation starts with the modelling of the optimisation subjects in order to understand the causal coherencies. That is the main reason for which each activity and service of the enterprise have been the object of modelling and formalisation for a lot of decades. According to the variety of activities, the number of models and of modelling techniques has been very increased over the years. The different services of the enterprise have been optimised, by using results of the computer science and the different theories of automatic control. It is now necessary to break the inharmoniousness of the separated application models to gain the productivity improvements. For this optimisation purpose other approaches, other theories and other tools are needed. The key words are therefore integration, computer integrated manufacturing, openness and open systems and interoperability.

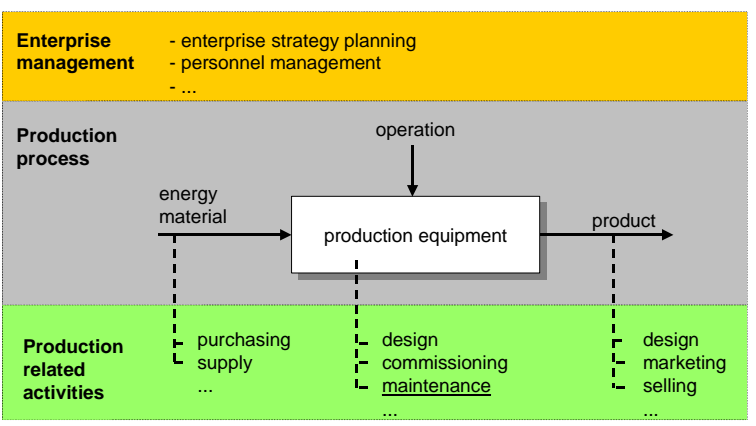

Figure 1. Elements of enterprise management

Some technologies have seriously contributed to this approach like various Internet technologies (HTTP, XML, SOAP, etc.), the STEP standard (ISO 10303), the OMG CORBA standard, the J2EE standards suite or also widely used proprietary technologies for distributed computing like Microsoft ${ }^{\circledR}$ DCOM. But 
also the decreasing cost of electronic and therefore the increasing number of controllers, computers and PLCs in all the production equipment have contributed.

All these technologies are necessary steps to an optimised maintenance management. But to reach the objective of global optimisation it needs more than just technology. It needs information models, which span different tools and which fit the complexity of the whole enterprise. These models have to integrate the heterogeneity of already existing application models. The model complexity and the need for integration of existing models make modelling difficult here.

Some modelling methods are of interest in this field like UML (Boch, 1999), (Rumbaugh, 1999), PERA (Williams, 1992), and GRAI (Doumeingts, 2000). They embed features of data modelling and of behavioural modelling for models of software applications.

The next step is the extension of all the activities of the enterprise using automatic control principles (the feedback and the closure of information loops, the scheduling) to all the activities of the enterprise. This is a trend in integration, which is among other reasons, the origin of the PROTEUS project, dedicated to the maintenance in industry.

The pant and equipment maintenance is a very important activity for all the industrial enterprises and for the improvement of product quality, performance of the plants, and satisfaction of the customers. Maintenance activities cover various operations of the enterprise management as

the plant and the equipment to be maintained (computer aided commissioning operation and diagnosis tools),

the management of human, material and financial resources,

the documentation management and further more.

Maintenance is therefore an activity, which needs the integration of several software applications associated to the different functions involved above. All these software sub-systems are currently based on different models, they are normally complementary, but sometimes redundant, incoherent and always heterogeneous.

The PROTEUS objective is therefore the integration of these various sub-systems by using a unique and coherent description of the equipment (through an information model description), a generic architecture (based on the "Web Services" technology) and coherent models of heterogeneous components.

By using this approach it becomes possible to access the equipment documentation in a unified way, independent of its origin, equipment manufacturer, integrator and end-user. Any operator or manager (obviously administered with the correct access rights) has the capability to remotely access relevant data from the SCADA or plant control system to request a diagnosis from a remote intelligent system, and therefore to prepare an intervention with optimality.

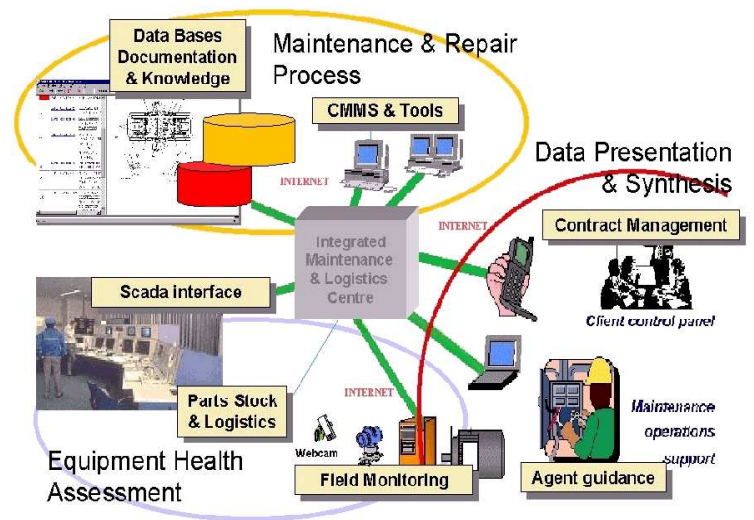

Figure 2. Overall maintenance components

Such a platform may be adapted to any production tool, in any domain (energy production in a nuclear power plant or by windmill, discrete part manufacturing companies, transport systems and so on.). The platform allows the outsourcing of maintenance to specialised companies, and the optimisation of different tasks or operations for the benefits of the different actors.

The platform structure is introduced in section 2. The components of the platform are described in section 3. Some modelling considerations related to the workflow are described in section 4 . Section 5 gives some conclusions.

\section{COMPOSITION OF A GLOBAL MAINTENANCE PLATFORM}

The PROTEUS platform is a system, which fits the needs of a maintenance platform as described in the previous section. Its architecture is illustrated in Figure 3. The PROTEUS platform is mainly focused on the infrastructure for integration rather than on the development of the dedicated maintenance tools.

Considering the market situation, diverse solutions are available for all the components arranged around the central operational core exiting a great range of applications. Also certain effort has been taken to realise data exchange between individual tools. In most cases this is limited to the point-to-point connection between individual tools following bilateral agreement for exchange formats. Initiatives like MIMOSA (Mimosa, 2004) have been established to unify data elements to be exchanged for special equipment like Condition Monitoring Tools or dedicated assets.

For the exchange of plant management data, powerful integration platforms, so called Enterprise Application Integration tools (like Biz Talk (BizTalk, 2004), TIB/Active Enterprise, e*Exchange, MQSerien Integrator, ETI EXTRACT, JARDiX Integration Suite, SCORE Integration Suite, eBusiness Integration (Nußdorfer, 2001)) have been designed to overcome the evil of diverse bilateral interface adaptation solutions. These solutions are cost intensive and mainly dedicated to B2B applications. 


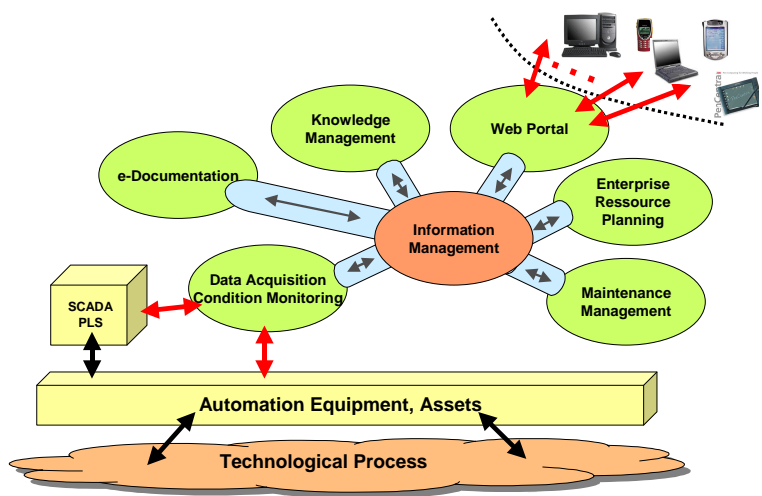

Figure 3. General maintenance platform components

The PROTEUS concept follows the idea of application integration but within the context of maintenance. Therefore it focuses on Maintenance Application Integration (MAI). It considers all those components delivering useful data for maintenance tasks:

- $\quad$ Enterprise Resource Planning (ERP) systems ERP tools like e.g. SAP/R3 are elementary parts of the enterprise management and are able to deliver information regarding assets or personal. Today's ERP systems may already contain modules for maintenance management.

- Maintenance Management Systems (CMMS) CMMS's are classically used to plan and control maintenance tasks.

- Knowledge management systems

Knowledge management methods are used to support diagnosis process as well as decisionmaking

- e-Documentation Servers

An e-documentation server is used for managing access to any kind of documentation used to describe assets or to support repair, inspection and servicing.

- Data Acquisition Servers

The availability of online data (status information) is the precondition for the application of condition based maintenance strategies. A Data Acquisition Server (DAS) shall be considered as a mean to deliver any kind of such data.

- Platform core applications

Several basic functions are needed to manage the co-operative operation of all the tools considered to be the elementary part of the eMaintenance platform. This mainly considers communication mechanisms, access right management, event distribution/notification, registration, scheduling of operations, work flow management.

An additionally introduced component is the WEB Portal. It integrates the applications on the presentation layer. The WEB Portal can be seen as the universal user interface to access all maintenance management related information. It is responsible for providing user input data to different types of client applications. In reverse direction it enables the human readability of data of the client applications.

It also defines means to instantiate business logic objects (BLO's). Business logic objects are used to control workflow as well as to transform data into or from a common exchange format used for communication within the PROTEUS platform. The consequent use of BLO's opens the way to real distributed design and execution of maintenance tasks while improving or even replacing centralised maintenance management through distributed components. The PROTEUS platform therefore can be considered as a distributed runtime environment for maintenance management applications.

The principal objective of the PROTEUS platform is to provide the means for moving from co-existence to interoperability and co-operation of these applications within the same environment. The interoperability should provide the condition for orchestration of components in order to provide a global integrated service for the platform user(s). The integration is necessarily done by the exchange of data between the components. This fact shows that the platform faces three principal structural constraints:

- Need for interoperability and co-operation of applications having a vast diversity of execution models: real-time for SCADA, transactional data access based for documentation and ERP, interactive (dialog based) execution for decision support tools

- Evident need for data exchange between remote sites, since the applications are rarely present on the same site

- Diversity of applications implies diversity of data formats and data volumes produced by them.

The concept of integration of services provides the service user with the comfort of allowing him a total abstraction of service implementation. User action is limited to the service invocation via publicly available interfaces. The system, which implements the whole framework takes in charge the identification of necessary components and the appropriate method of communication among them. The communication method would consist in choice of elementary services and their interfaces as well as the scheduling of service invocation and data exchange.

It becomes evident that the main issues of the integration platform construction are:

- Remote access to any component application, despite its position with respect to the requester, this implies the use of global networking facility such as Internet

- Efficiency of data transmission linked with the possibility of exchange of heterogeneous data formats 
- Distributed execution of component applications and independence of type of machine hosting an application

- Possibility of dynamic discovery of services, addresses and interfaces

- Possibility of programming of service chaining mechanisms

\section{PLATFORM INTEGRATION CONCEPT}

The PROTEUS project introduces a platform core, which enables information exchange and other interactions between different tools. This reduces the manual work, which is necessary for the data exchange. Moreover there evolves the possibility to get the tools synergetically working together. The PROTEUS platform demonstrates this feature on the area of plant and vehicular maintenance. In that case examples for tools are legacy applications like SCADA, ERP and CMMS and additional other applications developed within the project like documentation and data acquisition servers. The newly introduced synergetic abilities of the platform are provided via a WEB based human machine interface.

An adapter has to be used to connect a tool to the platform core. Within PROTEUS this adapter is called Intelligent Core Adapter. The wording "intelligent" reflects the characteristic of such an adapter to implement business logic rules.

A widely used model of software applications is that of a three-tier application. This model consists of the following tiers:

- Data tier

- Business logic tier

- Presentation tier.

Software becomes more and more complex. The model of a three-tier application has been introduced to distribute the software engineering work to specialists and special software components. Internally these tiers are mostly spread into sub tiers for enhanced modularisation.

A typical example installation of the PROTEUS platform is shown in Figure 4 (see also PROTEUS, D6.3).

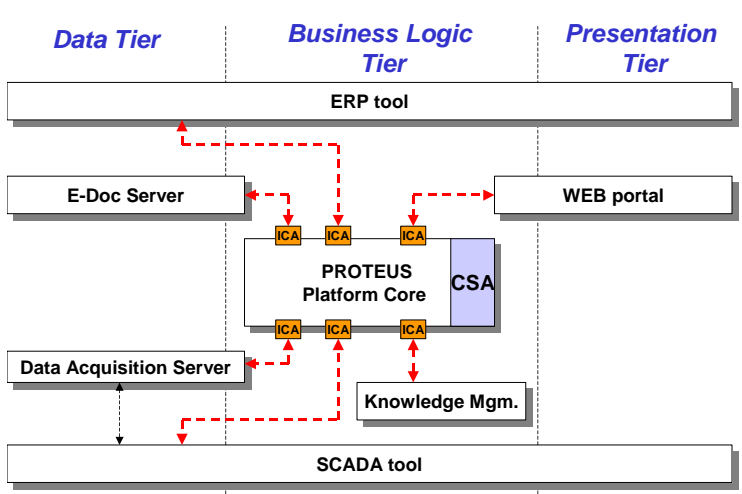

Figure 4. PROTEUS a three-tier platform
The Data Tier hosts all necessary information used for PROTEUS applications. Sources and destinations for process data, meta information about equipment and process management data are included in the Data Tier.

The Business Logic Tier is responsible for information processing. The raw data will be processed using business logic rules and algorithms for process monitoring and control either by human or other control algorithms.

There is a need for presentation of the interaction data for interaction between human and process control. This is done on the Presentation Tier.

Figure 4 shows that there exist applications in an enterprise, which provides all three tiers. Other applications are specialized systems of the one of the tiers. The PROTEUS Platform Core provides the possibility to the co-operative and orchestrated execution of distributed processes run on heterogeneous hardware/software platforms and communicated via Web Services.

The integration conecpt features three classes of elements, which are oriented to integration functionality:

- The Central Service Application (CSA) provide services for connection establishment and service authentication

- Intelligent Core Adapters (ICA) provide standardised interface transformers for the peripheral applications (platform tools)

- Functional Core Applications (FCA) are situated in the platform core and they implement supplementary functions needed by the global service requirements and not provided by the platform tools

The integration relies on a pool of central components, which allows applications to register themselves, discover other applications and forward the necessary descriptions of their interfaces. The pool of these components, called Central Service Application (CSA) is the heart of the PROTEUS platform. It represents the variety of component types; the non- exhaustive list is as follows:

- Tool interface register (UDDI registry),

- Work Flow Management tool,

- Security Manager (CAAS),

- Object Relation Database (CORD),

- Central Event Distribution Service (CEDS).

The key feature of the PROTEUS platform is the distribution over a local or global network. Following this principle the set of CSA components can be conveniently distributed over many execution supports for the overall system robustness and reliability. The roles of the key CSA components are briefly discussed below:

\section{UDDI server}

The existence of this component in the PROTEUS platform allows for the dynamic discovery of the services offered by the integrated applications. 
Dynamic discovery is a must in the case when different (i.e. not necessarily chosen a priori) platform tools can be integrated to the system.

\section{Work Flow management tool}

This is one of the key components providing the cooperation possibility among the different platform tools via synchronisation and orchestration of their operations. Its existence is dictated by the principle of integrated service creations.

\section{Security Manager}

The openness of the communication media exposes the platform to the external intrusions, be it non destructive or destructive. In principle all the components residing within the platform should be protected against unauthorised access. At the same time privileged communication channels should be granted to the flows, which contribute to cooperation and orchestration. In order to provide the adequate solution to the security issue, the global security policy for the particular installation has to be selected and implemented. This is the role of the security manager.

\section{Object Relation Database (CORD)}

The CORD provides functionality for definition and query of relations between data objects represented via the interfaces of ICAs. The idea of CORD follows the concept that the distributed tools and no central information database manage the platform data. The CORD manages only relations of data. For that purpose ICAs have to reflect the information model of the tool as object trees via a generic node interface. CORD is able to store pairs of object paths and a relation type reference per object relation. The CORD also manages the relation types. In that way CORD provides support for service driven cooperation of integrated applications.

\section{Central Event Distribution Service (CEDS)}

A complementary method of co-operation between distinct applications orchestrated in order to provide the integrated services is implemented by the mechanism of notification via events on the "subscriber - producer" principle. The task of event subscription and distribution is global and is implemented by a specialised central component.

\section{DESIGN AND IMPLEMENTATION OF WORK FLOW MANAGEMENT}

Figure 5 introduces an example workflow, which will be described in the following:

Certain equipment of an installation is monitored online to retrieve status information as a basis for condition based maintenance. In the case where a problem is detected, a message is sent to the CMMS, a trouble ticket is created and a notification is sent to the operator. The operator may retrieve additional information from the Data Acquisition Server as needed before requesting support from the expert system. The expert system tries to find a correction and to quantify the risk linked with the appearing problem. Therefore, it needs the knowledge database to have the description of the problem. Finally, it gets sensor values from the data acquisition server. If the evaluated risk is higher than a given bound, then the operator has to stop the process and repair. In this case it requires the e-doc server to have instructions to repair or documentation regarding equipment characteristics. Otherwise, an intervention is to be planned by the CMMS tool.

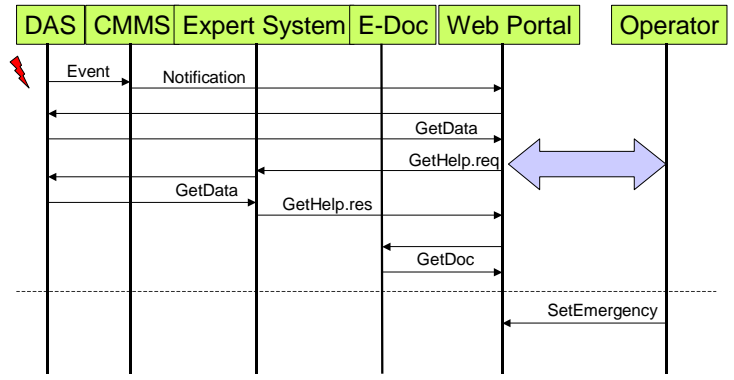

Figure 5. Work flow scenario

It becomes visible that the WEB Portal, the knowledge management tool, the Data Acquisition Server, the e-Documentation server and the CMMS are involved to realise this maintenance scenario.

The scenario is composed of several actions of actors involved and the control of information flow. The flow of information associated to business logic rules is managed by so-called BLO's (Business Logic Objects).

Business logic rules can be implemented by Intelligent Core Adapters (ICAs) and Functional Core Applications (FCAs). We distinguish between stateful and stateless business logic. One special case of a stateful BLO is a Workflow BLO. The following figure shows an example constellation on how the Workflow BLO interacts with other platform components.

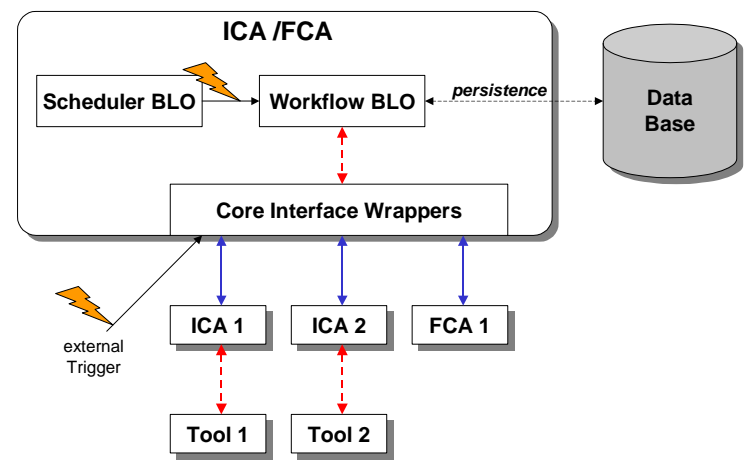

Figure 6. BLO interactions

The Intelligent Core Adapters have a persistence layer. This layer is responsible for the storage of the internal state of adapters. There are two possibilities to implement this persistence layer:

- Persistency will be provided by the adapter runtime environment (e.g. Microsoft BizzTalk server, J2EE server).

- Persistency will be managed by the adapter source code by saving the adapter state into a file or a database. There is no need for a complex and expensive runtime environment like in the 
first solution, however the amount of manual work for programming and testing increases dramatically.

Either an internal trigger like a scheduled event or an external trigger triggers the workflow. The Workflow BLO orchestrates different tools (via their ICAs) and FCAs once the workflow is started.

The workflow will be organised by finite or infinite state machines running in the Workflow BLO. A batch will be considered as a special finite state machine without internal loops. The actual state of the state machine will be persistent (stored in a database).

There are two main workflow types using the BLOs:

- Transactional workflows

- Interruptible workflows

The following figure maps the example given above to a sequence of a transactions between ICA's:

A trigger (detection of the problem) initiates the start of the state machine. This start is marked at the persistence system. Several interactions follow with the external ICA's (and optionally with FCA's) while the state machine steps from state to state. Finally all information related to this run of the state machine is deleted from the persistence store.

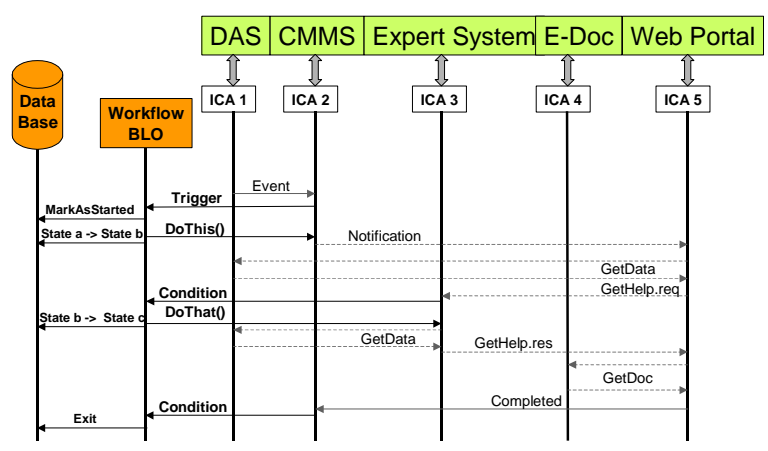

Figure 7. Work flow implementation

In the case of a transactional workflow, the state machine will run from its initial state after system restart if the system crashes where the Workflow BLO is running on.

Every state change and the according meta-results are persistently stored in the case of an interruptible workflow. This makes it possible to restart directly from the state, which was active before a system crash.

Assuming decades of maintenance scenarios there are multiplied numbers of state machines within BLO's. Additionally both scenarios and the behaviour of the BLO's have to be specified in a consistent way. The scenarios are the requirements, which have to be fulfilled by the BLO's. The result is that the specification has to be supported. Therefore formalised specification methods are applied for both aspects using UML sequence charts and state charts (for stateful BLO's) graphical languages. The UML meta model and its action semantic make it possible to check the consistency between sequence diagram and specified state machine. Several UML tools provide these checking means.

\section{SUMMARY AND CONCLUSION}

The paper describes the integration of busines logic objects in a platform to provide maintenance applications in enterprises. The structure and the mechansims are described. It becomes visible that the workflow is a key aspect of the maintenance application. Therefore special attention has to be drawn to its specification.

\section{ACKNOWLEDGEMENT}

This paper is based on the results and actual activities of the project PROTEUS sponsored by the French Ministry of Economy, Finance and Industry and the Federal Ministry of Education and Research of Germany under the label of European Commission Initiative ITEA (PROTEUS).

\section{REFERENCES}

Booch, G., Rumbaugh, J., Jacobson, I. (1999). The Unified Modelling Language User Guide, Addison-Wesley Longman.

Doumeingts G. et Al. (2000). Computers in Industry, Vol 42, 2000, pp.245-263.

INI (1987). The MAP Book: An introduction to Industrial Networking. Industrial Networking Incorporated, 3990 Freedom Circle, Santa Clara, CA 950528030 .

Rumbaugh, J., Jacobson, I., Booch, G. (1997). Unified Modelling Language Reference Manual, 12/1997.

Mimosa (2004). http://www.mimosa.org/osa-eai/.

BizTalk (2004). http://www.microsoft.com/biztalk/.

Nußdorfer, R. (2001). Das EAI-Buch, CSA Consulting $\mathrm{GmbH}$, http://www.nomina.de/eai/eaibuch.htm, 2001.

PROTEUS. A generic e-maintenance platform. http://www.proteus-iteaproject.com/, 2002

PROTEUS. D6.3 Definition/specification of objects for the e-maintenance platform. Intermediate report D6.3, September 2003 